\title{
Soft computing in remote sensing image processing
}

\author{
Yanfei Zhong ${ }^{1}$ - Zexuan Zhu' ${ }^{2}$ - Yew Soon Ong ${ }^{3}$
}

(C) Springer-Verlag Berlin Heidelberg 2016

Nowadays, more and more satellites with remote sensing sensors have been launched into the outer space, making remote sensing image one of the most important tools for decision-makers in a plethora of applications such as agriculture, mineralogy, physics, and surveillance. Nevertheless, the captured remote sensing images which characterized with high nonlinearity and high dimensionality have raised new challenges to practitioners in the area. In the recent years, soft computing techniques have attracted increasing attention in the computer science and engineering community due to its high capability in global optimization and handling nonlinear data, thus serving as a promising avenue for resolving some of the great challenges of remote sensing image processing.

We organized this special issue entitled Soft Computing in Remote Sensing Image Processing with the aim of bringing together researchers from both remote sensing and computational intelligence fields to showcase how soft computing techniques can be used or in tandem to help solve some of the challenges faced by practitioners in the remote sensing image processing area, while at the same time promoting advancements in this avenue. This special issue has received significant favorable responses from researchers that spanned all the globe.

Upon undergoing a thorough review process, only 9 most outstanding contributions have been accepted and included

Yanfei Zhong

zhongyanfei@whu.edu.cn

1 State Key Laboratory of Information Engineering in Surveying, Mapping, and Remote Sensing, Wuhan University, Wuhan, People's Republic of China

2 School of Computer Engineering, Nanyang Technological University, Singapore, Singapore

3 College of Computer Science and Software Engineering, Shenzhen University, Shenzhen, People's Republic of China in this special issue, covering applications of remote sensing image processing for panchromatic sharpening, endmember extraction, change detection, segmentation, band selection, unmixing, classification, and clustering. Particularly, the first paper of this special issue, entitled "Takagi-Sugeno Fuzzy System and MTF-based Panchromatic Sharpening", introduces a Takagi-Sugeno fuzzy to inject appropriate spatial details in the pansharpened image that is difficult to handle in traditional pansharpening methods.

The second paper entitled "A Quantitative and Comparative Analysis of Different Preprocessing Implementations of DPSO: A Robust Endmember Extraction Algorithm" compares different endmember candidates generation methods including PPI, VCA, N-FINDER for DPSO-based endmember extraction algorithm. The experimental results showed that VCA is the most reliable preprocessing implementation for DPSO.

The third contribution entitled "Difference Representation Learning Using Stacked Restricted Boltzmann Machines for Change Detection in SAR Images" established a deep neural network using stacked Restricted Boltzmann Machines to analyze the difference images and detect changes between multi-temporal synthetic aperture radar images, which included unsupervised layer-wise feature learning and supervised fine-tuning of network parameters.

The fourth paper entitled "Multispectral Remote Sensing Image Segmentation using Rival Penalized Controlled Competitive Learning and Fuzzy Entropy" proposed an image segmentation approach for multispectral remote sensing imagery based on rival penalized controlled competitive learning and fuzzy entropy, in which the cluster number can be determined automatically.

The fifth paper entitled "Band Selection for Hyperspectral Images Using Probabilistic Memetic Algorithm" presented an adaptive memetic algorithm for hyperspectral image band 
selection. The task of hyperspectral band selection was transformed into an optimization problem, where the balance between global and local optimization was achieved with an adaptive mechanism.

The sixth paper entitled "An Interpretable Fuzzy Monthly Rainfall Spatial Interpolation System for the Construction of Aerial Rainfall Maps" established an interpretable fuzzy model for monthly rainfall spatial interpolation by integrating the benefits of various soft computing techniques, which enabled researcher to gain greater insight into the spatial data to be modeled.

The seventh paper entitled "Adaptive Pixel Unmixing Based on a Fuzzy ARTMAP Neural Network with Selective Endmembers" applied a new selective endmember spectral mixture model to fuzzy ARTMAP neural network-based unmixing method, resolving the problem brought by the distinct forms of the endmember combination in diverse pixels.

The eighth paper entitled "Spatial-Spectral-Combined Sparse Representation-based Classification for Hyperspectral Imagery" suggested a new spectral-spatial sparse reconstruction classification method to fully take advantage of the spatial and spectral information in hyperspectral image by jointly considering the spectral and spatial neighborhood information of each pixel.

The final paper entitled "Multi-Objective Semi-Supervised Clustering for Automatic Pixel Classification from Remote Sensing Imagery" proposed a semi-supervised clustering methods for remote sensing image, in which Fuzzy C-means clustering method is used to generate labeled data based on the unlabeled remote sensing data. Subsequently, archived multi-objective simulated annealing-based technique is then employed as the framework to optimize all three objective functions simultaneously.

The papers collected in this special issue have reflected the present state-of-the-art advancements of soft computing in remote sensing image processing. Thus we believe that the papers published in this issue will also serve to identify new open research issues, intriguing challenges and opportunities of soft computing in remote sensing image processing.

We would like to gratefully acknowledge and sincerely thank all the reviewers for their insightful comments and criticism of the manuscripts. In addition, we would also would like to thank the authors for their great contributions to this special issue. Finally, we are grateful to the Editor-in-chiefs Antonio di Nola and Vincenzo Loia and the editorial office assistants of Soft Computing for their immense support during the review and production process of this special issue.

\section{Compliance with ethical standards}

Conflict of interest The authors declare that there is no conflict of interests regarding the publication of this paper. 